\title{
Importance of long term follow up in pheochromocytoma
}

Bellarbi D, Azzoug S, Rabehi L, Chentli F

Endocrine and Metabolic diseases department Bab El Oued Hospital Algiers Algeria

\section{INTRODUCTION}

Pheochromocytomas and paragangliomas are rare but life threatening tumors secreting catecholamines. They could be either sporadic or inherited. Either isolated or integrated in genetic syndromes such as Von Hippel Lindau (VHL) (OMIM193300) which is an autosomal dominant disorder resulting from germline mutations in the VHL gene.

We report a 42 year old man operated at 8 years old for bilateral pheochromocytoma revealed by adrenergic symptoms and high blood pressure. Unfortunately, at that period he did not have any genetic testing. However, there were not clinical clues for a genetic syndrome. At the age of 42 , he had neurological manifestations and chronic diarrhea.

Clinical examination showed a static cerebellar syndrome and central vestibular syndrome.

Cerebral MRI showed supra and infra-tentorial lesions deemed to be hemangioblastomas. The largest lesion was located in the left cerebello-protuberancial area and measured $37 \times 45 \times 33 \mathrm{~mm}$ (Fig 1) for which he underwent neurosurgery. Pathology examination confirmed the diagnosis of cerebellar haemangioblastoma. Abdominal Computed Tomography (CT) revealed 03 pancreatic neuroendocrine tumors (NET) and a $12 \mathrm{~mm}$ mesenteric nodule (Fig 2) presenting as a homogenously and typical NET. The largest pancreatic NET had intensive fixation at octreoscan (Fig 3). Ophthalmologic examination revealed retinal hemangioblastoma. The diagnosis of VHL was therefore clinically made. Familial investigation revealed the same syndromic VHL in one sibling who had bilateral pheochromocytoma in his infancy and who is now followed for retinal hemangioblastomas associated to cerebellar hemangioblastomas. Genetic testing would be extremely useful to determine which individuals from his family are harboring the mutated allele.

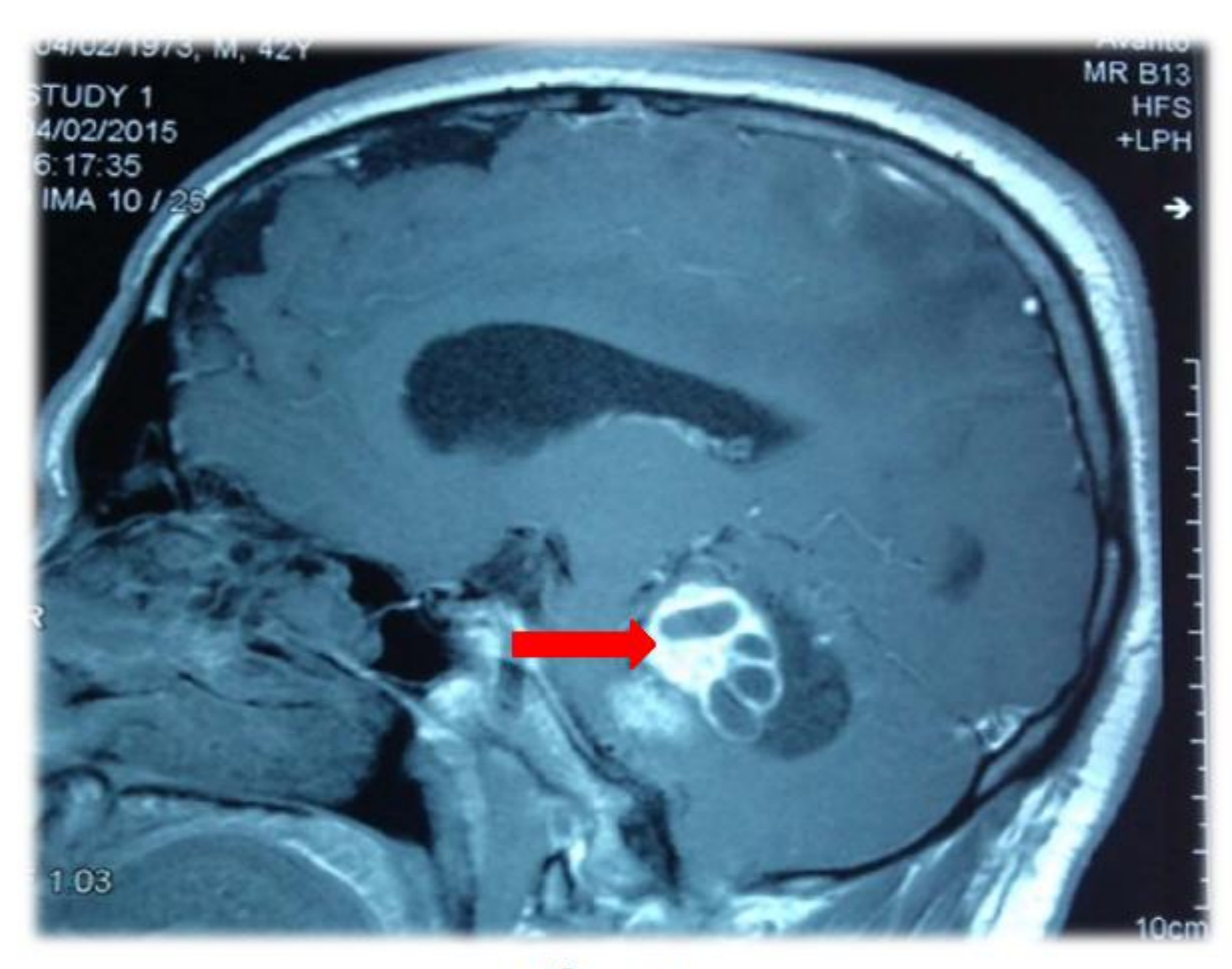

Fig 1

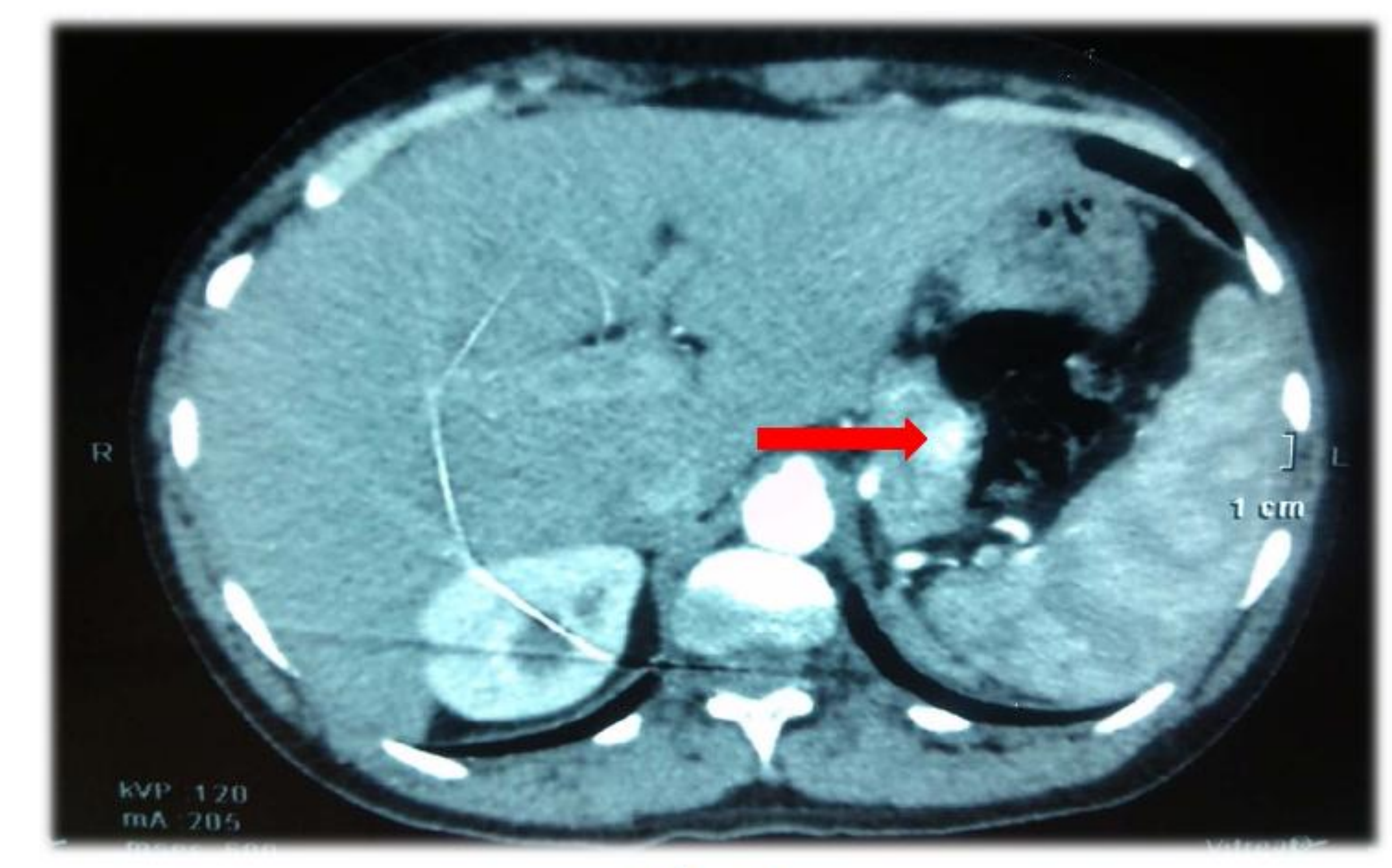

Fig 2

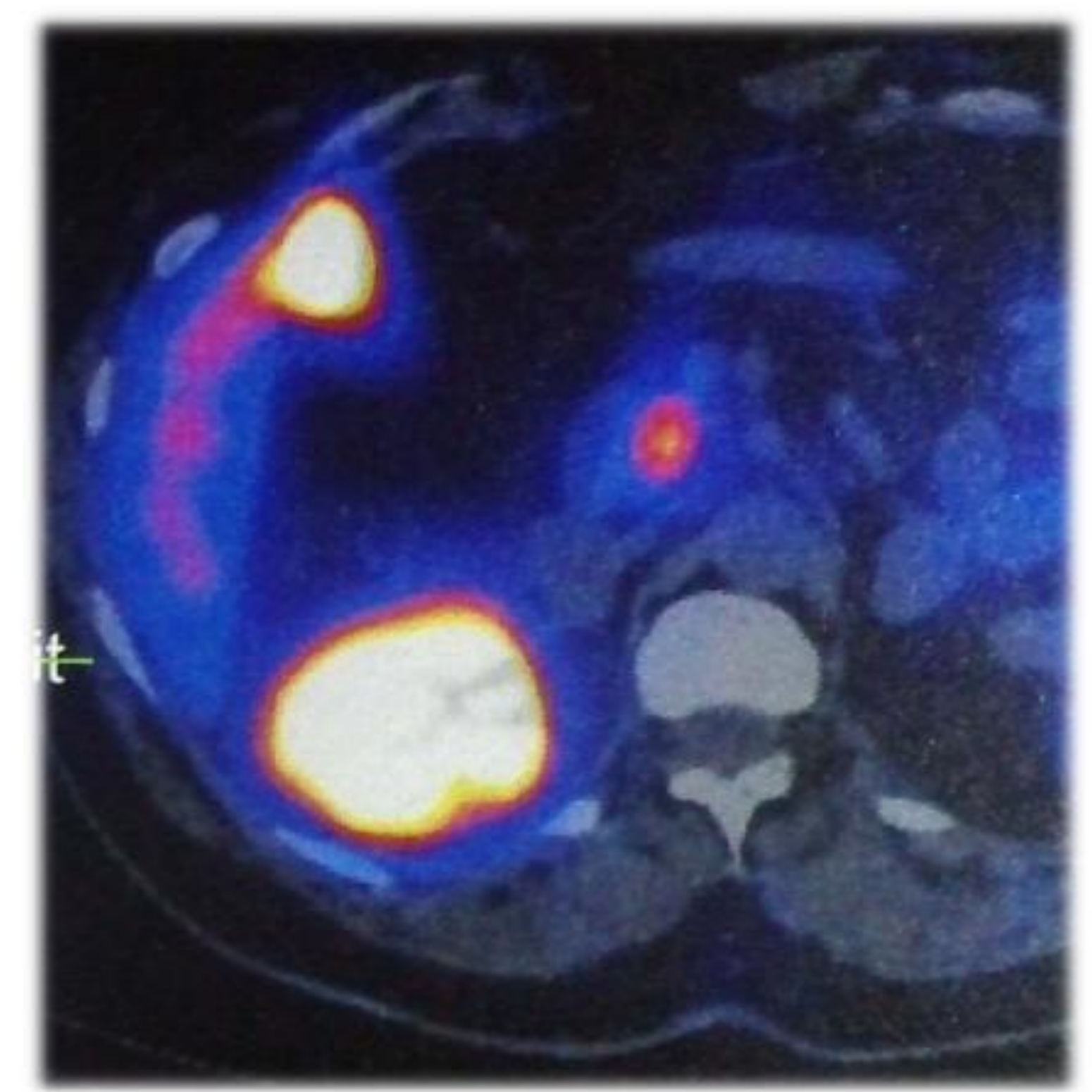

Fig 3

\section{CONCLUSION}

Patients operated on for pheochromocytoma especially young ones should have genetic screening in order to diagnose genetic syndromes which require a specific and a multidisciplinary approach for the diagnosis and treatment of all associated tumors and lesions. 